\title{
Splints in Dentistry- A Contemporary Review
}

\author{
Avinash Gohilot ${ }^{1}$ and Shikha Rastogi Gupta ${ }^{2 *}$ \\ ${ }^{1}$ Specialist Orthodontist, Vurjeel Medical Centre, Abu Dhabi, UAE \\ ${ }^{2}$ Specialist Orthodontist, Tooth Health Dental Centre, Agra, Uttar Pradesh, India \\ *Corresponding Author: Shikha Rastogi Gupta, Specialist Orthodontist, Tooth \\ Health Dental Centre, Agra, Uttar Pradesh, India.
}

Received: January 06, 2022

Published: January 25, 2022

C) All rights are reserved by Avinash

Gohilot and Shikha Rastogi Gupta.

\section{Abstract}

The ultimate goal in successful management of mobile teeth is to restore function and comfort by establishing a stable occlusion that promotes tooth retention and the maintenance of periodontium and temporomandibular joint health. The clinical management of mobile teeth can be a perplexing problem, especially if the underlying causes for that mobility have not been properly diagnosed. In some cases, mobile teeth are retained because patients decline multidisciplinary treatment that might otherwise e also include strategic extractions. Some mobile teeth can be treated through occlusal equilibration alone (primary occlusal trauma) whereas mobile teeth with a compromised periodontium can be stabilized with the aid of provisional and/or definitive splinting (secondary occlusal trauma). This review discusses splinting and its, rationale, indications, limitations and different types of tooth splinting are also reviewed.

keywords: Splints; Stabilization Splint; Temporary Splint; Provisional Splint; Permanent Splint; Periodontium; Temporomandibular Joint

\section{Introduction}

A Splint is a rigid form of support used to immobilize and protect a part of the body.

Splint is usually made of hard acrylic that fits over the occlusal and incisal surfaces of the teeth in one arch, creating precise occlusal contact with the teeth of the opposing arch. It is commonly referred to as a bite guard, night guard, interocclusal appliance, or orthopedic device [1].

\section{Objectives of splinting}

The various purpose of doing splinting is rest is created for the supporting tissues giving them a favorable climate for repair of trauma. It helps to stabilize the teeth during and after orthognathic surgery so that they introduce an optimum functional occlusion that reorganizes neuromuscular reflex activity which in turn reduces abnormal muscle activity while encouraging more normal muscle function. It helps to stabilize mobile teeth during regenerative periodontal therapy and protect teeth along with supportive structures from abnormal structures from abnormal forces that may create breakdown or tooth wear. Discomfort and pain are eliminated in temporo mandibular disorders and make the teeth conform to a correct skeleton related position of the condylar axis and verify that the correct position and alignment of the condyle disk assemblies have been achieved.

\section{Types of splint [2]}

- $\quad$ Stabilization splint: Is an increase in resistance to applied force by providing reciprocal antagonisms and increasing the effective root area. The force may remain the same, but the resistance is increased.

- $\quad$ Temporary splint: Is used on a short-term basis, usually less than 6 months, and is often advocated to stabilize teeth during periodontal treatment. It may or may not 1 ead to other types of splinting. 
- Provisional splint: Is used for a longer period of time from several months to as long as several years. It is used for diagnostic purposes, allows the clinician to see how teeth will respond to treatment and leads to more permanent forms of stabilization.

- $\quad$ Permanent splint: Also referred to as Periodontal prosthesis may be defined as those restorative and prosthetic endeavors that are indicated and essential in the total treatment of advanced periodontal disease.

\section{Various splints used in dentistry}

\section{Splint used in periodontally compromised dentition}

Wire ligation [3] is the most commonly used type of stabilization. Wire used is 0.008 " stainless steel. It is easy to construct. However, one of its basic limitations is that it can be utilized only where coronal form permits, Because of this shortcoming it has its greatest use in stabilizing the mandibular incisors. After an interproximal tie is made, connecting the buccal and lingual segments of the mesh, tooth-colored, self-curing acrylic is painted over the wire to obtain a more pleasing aesthetic result.
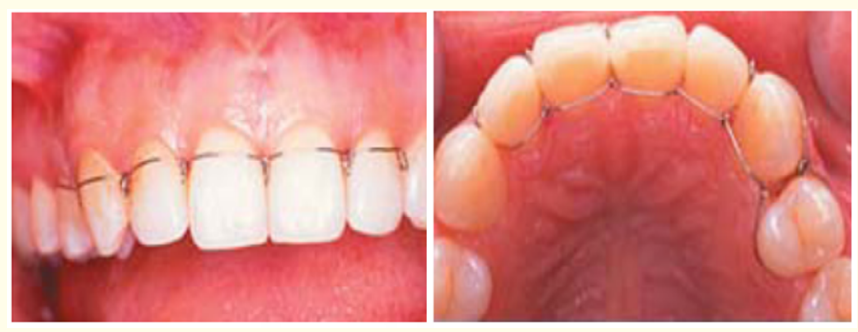

Figure a

Wire and acrylic: (A - splint) [3]

Obin and Arvins have described a technique of stabilization whereby wire (usually twisted in the form of a braid) is fixed with acrylic into channels prepared in mobile teeth. This approach can be utilized on the occlusal surfaces of posterior teeth and the lingual surfaces of anterior teeth. This technique offers advantages over the other forms of stabilization because there is greater control over coronal forms, occlusion, embrasure areas, and aesthetics. Acrylic internal temporary splints (A splints) require the preparation of a channel approximately $3 \mathrm{~mm}$ wide and $2 \mathrm{~mm}$ deep in several teeth. The preparations should be slightly undercut for re- tention. The pulpal surfaces should be coated with a protectant. A piece of platinized knurled wire 22 to 16 gauge $(0.64$ to $1.3 \mathrm{~mm}$ in diameter) is placed in the channel. Then self-cure acrylic is placed to fix the wire in the channel. Adjust the occlusion and polish the splint. This approach can be utilized more readily with anterior teeth. Disadvantage is the possibility of recurrent caries

\section{Composite resin splints [4]}

Restorative materials that are polymerized by ultraviolet light are very useful in providing stabilization of excessively mobile teeth. Basically, the technique is a simple one and provides adequate stabilization if care is taken during the actual operative procedures.

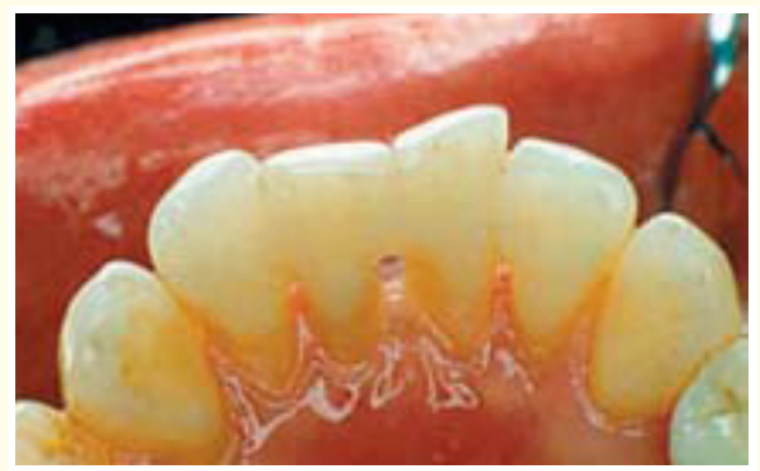

Figure b

The composite resin splint can be strengthened by adding wire, monofilament line, fiberglass or by using a fibre meshwork (e.g., Ribbond, Ribbond Inc., Seattle, WA) to reinforce the material.

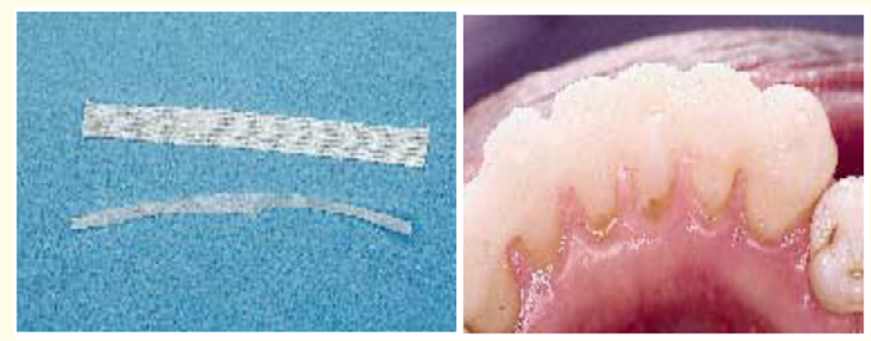

Figure c 
Fixed lingual splint [4]

Use of 0.0215-inch multistrand wire or $0.030-0.032$ inch sandblasted round stainless-steel wire can be adapted onto the lingual surface and stablized with composite can be used stabilization of mobile. Advantges: esthetics, ease of placement, non invasive, oral hygiene can be maintained.
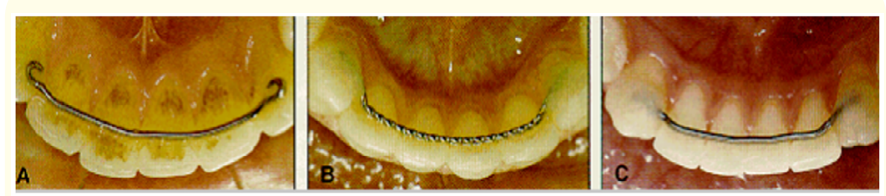

Figure d

\section{Acrylic bite guards (Night Guards) [5]}

They are made up of either acrylic or polyvinyl material used in the treatment of bruxism and clenching. The most common type of appliance is one that covers the occlusal surfaces of the teeth. For additional support the palate is often covered. An important consideration with all these appliances is that they must not obliterate the interocclusal distance (free-way space).

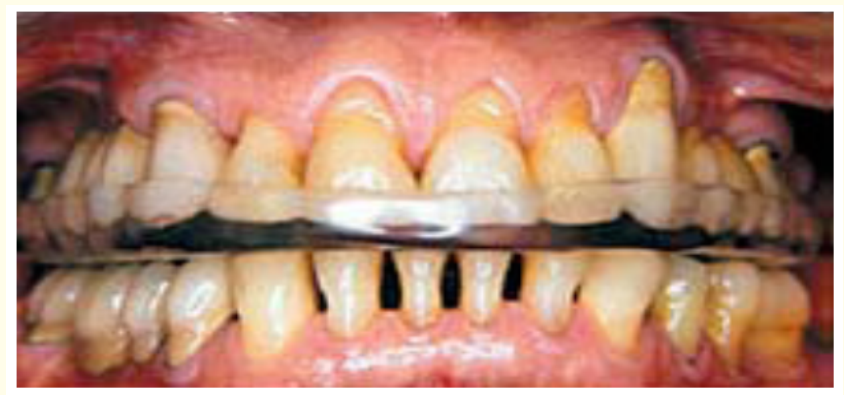

Figure e

\section{Removable cast appliances [5]}

It is usually a rigid casting either of gold or chrome cobalt, made to fit around the teeth. One end usually the anterior section, is not joined but is left open so that the casting can be sprung over the undercuts and then ligated.

The posterior end is continuous from the buccal to the lingual surface and is distal to the most posterior tooth. Another modification is an interlocking attachment on the distal end so that the ap- pliance can be locked after being sprung over the teeth. Obviously, with any form of removable splint, it is only effective if the patient wears the appliance.

\section{Extracoronal resin-bonded retainers [5]}

They can be fabricated in the dental laboratory, serve to strengthen the overall bonded situation.

The splints are usually cast from metals, usually non noble alloys that can be electrolytically or chemically etched or composite material. This type of splint has greater inherent strength than a composite-resin splint created intraorally.

Extra features such as grooves, pins and parallel preparations increase the retentive capacity of these splints.

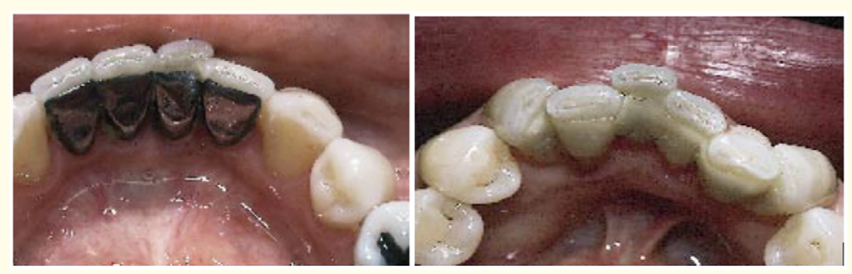

Figure $\mathbf{f}$

\section{Splints for TMJ dysfunction}

\section{Stabilization appliances [6]}

Sometimes called muscle relaxation appliance, because it is primarily used to reduce muscle pain. It is generally fabricated for the maxillary arch and provides an optimum functional occlusion for the patient. When the appliance is in place, the condyles are in their most musculoskeletal stable position at the time that the teeth are contacting evenly and simultaneously. The treatment goal of the stabilization splint is to eliminate any orthopedic instability between the occlusal position and the joint position, thus removing this instability as an etiologic factor in the TMD. Stabilization splints are full coverage hard acrylic resin appliances that can be constructed to fit on either the maxillary or mandibular arch. The various indications are treatment of masticatory dysfunction signs and symptoms such as muscular pain, TMJ pain, clicking, crepitus, limitation of motion, incoordination of movement, Bruxism, Local muscle soreness or chronic centrally mediated myalgia and $\mathrm{Pa}-$ tients experiencing retro discitis. 


\section{Canine protected splint [6]}

A splint core fabricated from a single sheet of $1.5 \mathrm{~mm}$ thermoplastic hard acrylic, with ball end clasps between molars and premolars for retention. The splint core was placed in the patients mouth and acrylic was added to produce a hard-anterior flat plane stop, with minimal separation of posterior teeth. Soft acrylic was added posteriorly and a centric relation bite was obtained with the patient closing until contacting the anterior flat plane. Additional acrylic was added in the canine region to limit lateral movement. It has to have Full time wear. The splint was found to be effective in limiting the lateral movements so that the patient opens vertically. There is immediate posterior disocclusion in all mandibular movements. An immediate complete anterior guidance development (ICAGD) occurred which is effective in the treatment of MPD. Splint works by prevention of lateral movements, which reduces loading in the TMJ and reduction in parafunctional activity.

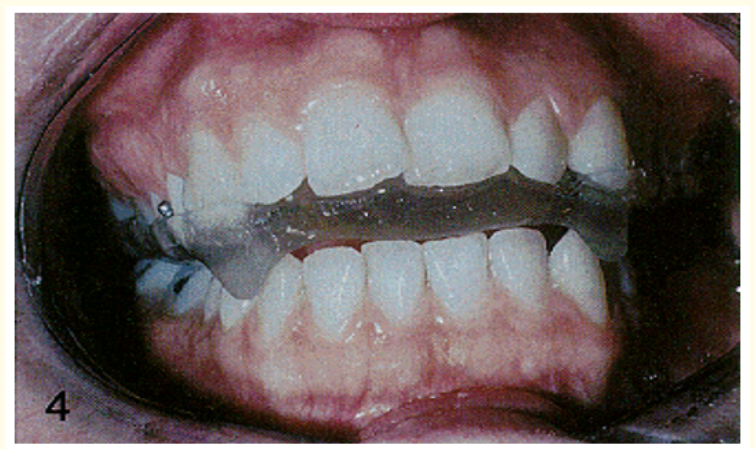

Figure $\mathbf{g}$

\section{Anterior bite plane [6]}

The anterior bite plane is a hard acrylic appliance worn over the maxillary teeth, providing contact with only the mandibular anterior teeth. It is primarily intended to disengage the posterior teeth and thus eliminate their influence on the function of the masticatory system.

The various indications include treatment of muscle disorder related to orthopedic instability or an acute change in the occlusal condition, Parafunctional habits and used overnight as muscle deprogrammer.

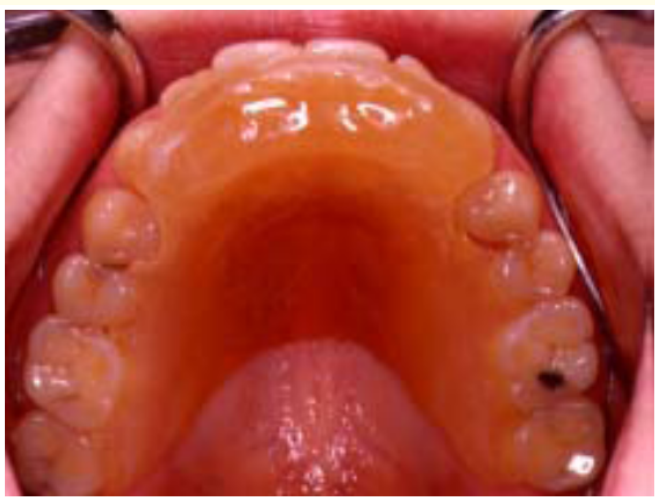

Figure h

\section{Pivot splint [6]}

The pivoting appliance is a hard splint that covers one arch and usually provides a single posterior contact in each quadrant. It is used to treat non reducing anterior displacement of the meniscus. When superior force is applied under the chin, the tendency is to push the anterior teeth close together and pivot the condyles downwards around the posterior pivoting point.

Unilateral pivot splint distracts the condyle from the fossa. When a unilateral pivot is placed in the second molar region, closing the mandible on it loads the contralateral joints and slightly distracts the ipsilateral one. This device should not be used for longer than 1 week, since it is likely to intrude the second molar.

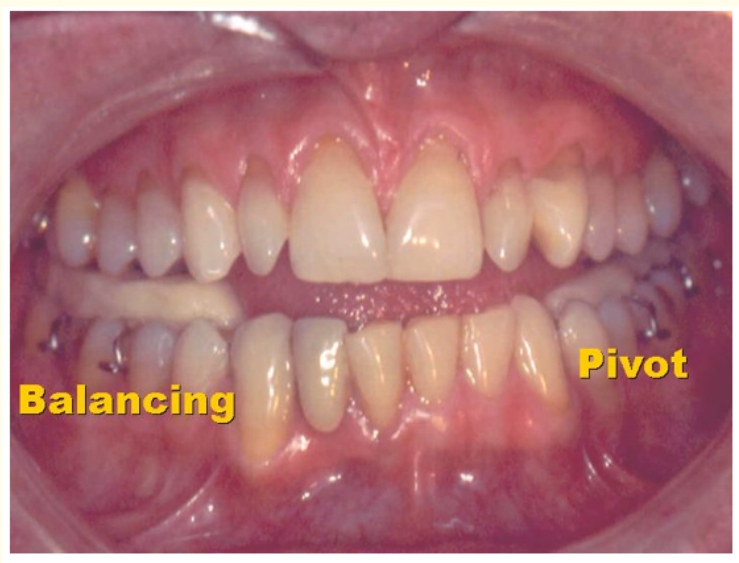

Figure i 
Glass ionomer orthodontic pivot splints [6]

A pivot splint can be constructed by filling the fossa of the lower first molars with a glass ionomer cement. It disocclude all other teeth, bringing the mandible in centric relation. Four points of maxillomandibular contacts occur in centric relation. Two temporomandibular articular discs with the condylar articular surfaces, and the two maxillary first molar mesiolingual cusps with the glass ionomer pads on the maxillary first molars. In lateral motion, these are 3 points of contact i.e., a balanced triangle formed by the two condyles and one glass ionomer pad.

All the teeth, including second molars are bracketed. The splint is particularly useful in cases where a cusp tip must be jumped past an opposing cusp tip. With the load shared by all the teeth through the arch wire, the splint allows the benefits of 'deprogramming' and vertical opening while the teeth are moved.

The various indications are Cases with deep overbite and advanced attrition of incisal and cusp tips. Glass ionomer splint allows immediate placement of full fixed appliances while opening bite and establishing centric relation.

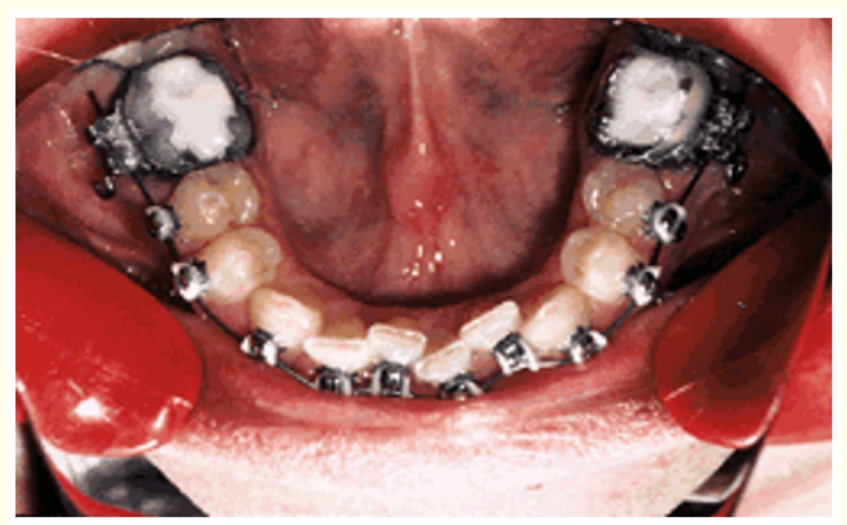

Figure j

Splint used for bi jaw orthognathic surgery [7]

Made up of cold cure acyrlic with minimum thickness of $2 \mathrm{~mm}$ during pre surgical phase placed between the upper and lower jaw during bi jaw surgey for their stabization.

Disadvantages of splint [8,9] may be grouped under two categories i.e., Hygienic in which all splints hamper the patient self-care.

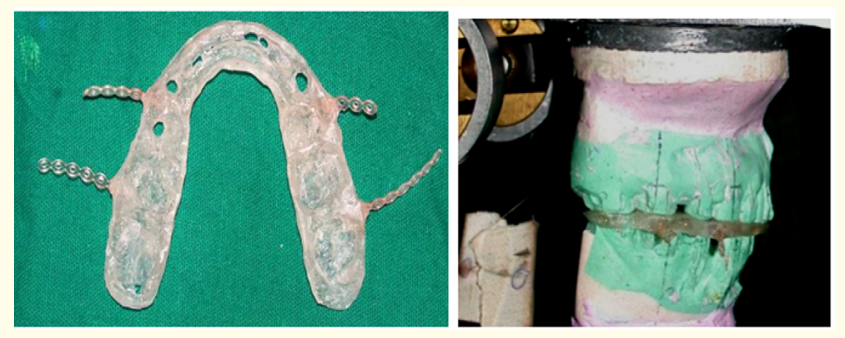

Figure k

Accumulation of plaque at the splinted margins can lead to further periodontal breakdown in a patient with already compromised periodontal support. It is also difficult to achieve proper contour of a splint at the gingival margin, especially in the interproximal areas. The second category is Biological development of caries is an unavoidable risk. It requires excellent maintenance by the patient. Splints, especially full coverage splints, may allow the development of extensive caries under loose abutments, without symptoms. It is very important that splints be inspected regularly and that that patient be examined for the development of caries. Splints should never be used as a "shotgun" substitute for accuracy and precision.

\section{Conclusion}

The single observation of tooth mobility is not unto itself sufficient justification to splint teeth. Tooth mobility alone does not necessarily indicate the existence of an underlying pathologic condition. Splinting teeth to each other allows weakened teeth to gain support from neighboring ones. Splinting is best viewed as a preventive treatment measure for teeth that have minimal or no bone loss yet are clinically mobile. However, splinting makes oral hygiene procedures difficult.

Therefore, to ensure the longevity of the connected teeth, special attention must be given to instructing the patient about enhanced measures for oral hygiene after placement of the prosthesis. Splinting affords no guarantee that occlusal stress can be completely eliminated. Before treatment is started, it is recommended that the cause of any mobility be identified to determine if it is related to an occlusal discrepancy. It may be that an occlusal equilibration and splinting (provisional or definitive) may actually prevent tooth loss and restore both patient comfort and function. 
Thus "splinting may serve as a boon, improving the health of the periodontium and temporomandibular joint thereby decreasing tooth mobility and occlusal disturbances".

\section{Bibliography}

1. Carranza Jr FA. "Glickmanns Clinical Periodontology $6^{\text {th }}$ ed". Philadelphia. W.B. Saunders co (1984).

2. Lemmermans K. "Rationale for stabilization". Journal of the American Dental Association 31 (1950): 217.

3. Clark J., et al. "The wire ligature acrylic resin splint". Journal of Periodontology 40 (1969): 371.

4. Poison AM and Billen JR. "Temporary splinting of teeth using ultraviolet polymerized bonding materials". Journal of the American Dental Association 89 (1974): 1137-1141.

5. Watkins SJ and Hemmings KW. "Periodontal splinting in general practice”. Dental update 34(2000):168-180.

6. Hirschfeld L. "The use of wire and silk ligatures". Journal of the American Dental Association 31 (1950): 217.

7. Jeffrey P Okeson. "Fundamentals of occlusion and temporomandibular disorders" (1985).

8. Proffitt WR and White RP. "Surgical Orthodontic Treatment St Louis, Mosby" (1991).

9. Fermin A Carranza. "Clinical Periodontology ( $6^{\text {th }}$ edition)”.

\section{Assets from publication with us}

- Prompt Acknowledgement after receiving the article

- Thorough Double blinded peer review

- Rapid Publication

- Issue of Publication Certificate

- High visibility of your Published work

Website: www.actascientific.com/

Submit Article: www.actascientific.com/submission.php Email us: editor@actascientific.com

Contact us: +919182824667

Citation: Avinash Gohilot and Shikha Rastogi Gupta. "Splints in Dentistry- A Contemporary Review". Acta Scientific Dental Sciences 6.2 (2022): $112-117$. 